\title{
The survival and transport to the site of fertilization of diploid rabbit spermatozoa
}

\author{
D. Mortimer \\ Department of Genetics, University of Edinburgh, \\ King's Buildings, West Mains Road, Edinburgh EH9 3JN, U.K.
}

\begin{abstract}
Summary. The possible involvement of diploid spermatozoa in the production of triploid zygotes in the rabbit has been investigated in terms of the success of diploids relative to haploids in reaching the site of fertilization, with special reference to any possible selectivity of sperm transport within the female tract. The observed large significant reduction in the incidence of diploid spermatozoa between the ejaculate and the oviducts would greatly reduce the chances of, but not necessarily prevent, a diploid spermatozoon fertilizing an egg. This reduction appears to be due to a combination of the reduced survival of diploids in the female tract and a selectivity of sperm transport dependent on sperm motility.
\end{abstract}

\section{Introduction}

From observations on the mean head area and DNA content of ejaculated rabbit spermatozoa (Beatty \& Fechheimer, 1972; Carothers \& Beatty, 1975) it has been shown that individual haploid and diploid spermatozoa can be recognized with a high degree of confidence by simple visual appraisal of head size.

If a diploid spermatozoon were to fertilize a normal oocyte then the resulting triploid embryo would, presumably, be non-viable. However, such triploids as are found in the rabbit are most probably due to chromosome doubling in the oocyte rather than to fertilization by diploid spermatozoa (Fechheimer \& Beatty, 1974; Beatty, 1974). Therefore diploid rabbit spermatozoa would be expected to have reduced fertility relative to normal haploid spermatozoa because of one or more of the following reasons. Diploid spermatozoa may be dead or dying when ejaculated, or they may not survive as long as do haploid spermatozoa after ejaculation. The motility of diploid spermatozoa may be impaired or totally absent, resulting in poor penetration of the cervical mucus. Diploid and other abnormal spermatozoa may be 'filtered out' by the cervix, or the transport of diploid spermatozoa in the uteri and oviducts may be impaired or selectively inhibited because of their larger size. Physiological mechanisms of the female tract may actively 'persecute' diploid spermatozoa because of their abnormality. Finally, even if they reach the site of fertilization, diploid spermatozoa may be unable to capacitate or to undergo, or participate in, one or more of the various stages of the complex fertilization process.

The present study was designed to determine whether diploid rabbit spermatozoa are as successful as haploids in reaching the site of fertilization, with special reference to any possible selectivity of sperm transport within the female reproductive tract. The intrinsic fertilizing ability of diploid spermatozoa will not be considered at the present time.

\section{Materials and Methods}

The highest recorded natural incidence of diploid spermatozoa in rabbits (about $1.6 \%$ ) was found in young (20-40-week-old) bucks of the AD strain of dark chinchilla rabbits maintained at the Edinburgh Institute of Animal Genetics (Beatty \& Fechheimer, 1972). The particular buck used in the present work commonly produced about $2 \%$ and, on occasions, as many as $2 \cdot 3 \%$ diploid spermatozoa. 


\section{Ejaculated spermatozoa}

Ejaculates were collected by artificial vagina and immediately split into two parts. The first part was diluted at once with an equal volume of formol citrate (Dott \& Foster, 1975) and later further diluted (with the same diluent) to obtain a concentration of spermatozoa suitable for making good nigrosin-eosin smears. These were prepared by adding an equal volume of nigrosin-eosin ( $2 \mathrm{~g}$ eosin $\mathrm{Y}$ in $120 \mathrm{ml} \mathrm{10 \%} \mathrm{nigrosin} \mathrm{refluxed} \mathrm{for} 8 \mathrm{~h}$ ) to the diluted semen and leaving the mixture for $5 \mathrm{~min}$ at room temperature (about $22^{\circ} \mathrm{C}$ ) before smearing on clean microscope slides. The smears were allowed to dry in air and subsequently mounted with DPX. The second part of each ejaculate was used for evaluation of the semen by subjective estimates of sperm density and motility. From each ejaculate judged as 'good' (i.e. having a high sperm density and a high percentage of progressively motile spermatozoa) 10,000 spermatozoa were classed as haploid (whether live or dead), diploid live or diploid dead, and in a separate sampling 1000 haploid spermatozoa were classed as live or dead. In all classifications, spermatozoa showing any degree of eosinophilia were classed as 'dead' and unstained spermatozoa were classed as 'live'.

\section{Survival of ejaculated spermatozoa in vitro}

Immediately after collection, one drop of an ejaculate was diluted with 3 drops of formol citrate and used to make nigrosin-eosin smears (as described above). The remainder was divided into three

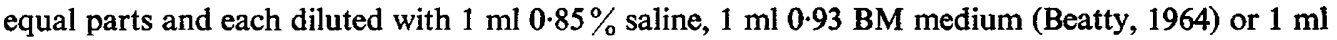
Hank's BSS and incubated at $37^{\circ} \mathrm{C}$ in sealed glass vials. Samples of $0.1 \mathrm{ml}$ were taken from each vial after incubation for $0,1,2,3,4,5,6$ and $7 \mathrm{~h}$, diluted with $1 \mathrm{ml}$ formol citrate and centrifuged at $500 \mathrm{~g}$ for $10 \mathrm{~min}$. After resuspension in 2 drops of formol citrate, nigrosin-eosin smears were made of the spermatozoa.

Two smears were made from each sample and after coding and randomization of all slides they were scored under oil immersion (at a magnification of $\times 1250$ ) for the percentage of eosinophilic spermatozoa in counts of haploid and diploid cells ( 100 of each per slide).

\section{Spermatozoa recovered from the female tract}

In each of 9 experiments semen was collected from the chosen buck and approximately $\frac{3}{4} \mathrm{~h}$ later the buck was mated with a receptive doe. A further ejaculate was collected about $\frac{3}{4} \mathrm{~h}$ later for comparison with that collected before mating. This frequency of ejaculation may have caused some temporary depletion of the buck's sperm reserves, but there were no significant differences in the proportion of diploid spermatozoa in the ejaculates collected before and after coitus. Six hours after coitus the doe was killed by an overdose of Nembutal (Expiral: Abbott Laboratories Ltd, Queenborough, Kent) injected into the ear vein. The abdominal cavity was immediately opened and the reproductive tract divided into compartments with artery forceps. The uteri were isolated from the oviducts within 5 min of death. There was no evidence of the "uterine pumping" described by Cohen $\&$ Werrett (1975). After excision the female tract was placed in mammalian Ringer-Locke saline at $37^{\circ} \mathrm{C}$.

The tract was dissected free of adnexa and divided into its various sections which were then flushed with either culture medium (Eagle's Medium with Earle's Balanced Salt Solution and HEPES buffer, Flow Labs) or phosphate-buffered saline (PBS Tablets: Oxoid) at $37^{\circ} \mathrm{C}$. The flushing media had a small amount of detergent added (1 drop of $1: 5000$ 'Teepol' per $2 \mathrm{ml}$; see Braden, 1953) to improve sperm recovery. The flushings were centrifuged in siliconized tubes at $500 \mathrm{~g}$ for $20 \mathrm{~min}$ and the spermatozoa resuspended in $2 \mathrm{ml}$ formol citrate. The suspensions from the four regions of the female tract were then centrifuged again and resuspended in formol citrate: 3 drops were used for the uterine and cervical spermatozoa, and the vaginal spermatozoa were diluted to a suitable concentration so that after smearing the spermatozoa were not overcrowded. Five smears of each of these sperm suspensions were made on clean glass microslides and allowed to dry in air before fixation and staining (Casarett, 1953). The small volume of suspension left in each tube was used for 
nigrosin-eosin preparations (see above). Oviductal spermatozoa were resuspended in 2 drops of distilled water to burst contaminating red blood cells and then placed under coverslips with vaselined edges and scored by phase-contrast microscopy.

Ten thousand spermatozoa ( 2000 from each of the 5 slides) from each of the pre- and post-coital ejaculates and the vaginal, cervical and uterine levels of the female tract, and also all of the spermatozoa recovered from the oviducts were scored at a magnification of $\times 500$ for the percentage diploid. Diploid spermatozoa were identified by their larger and more pear-shaped heads (see Beatty \& Fechheimer, 1972; Carothers \& Beatty, 1975). There was no variation in the identification of diploids whether scored in nigrosin-eosin, Casarett or phase-contrast preparations.

\section{Results}

\section{Ejaculated spermatozoa}

The percentages of dead haploid and diploid spermatozoa in ejaculates subjectively judged as 'good' (see Materials and Methods), are shown in Table 1. It is obvious from the invariably negative differences that more diploid than haploid spermatozoa were dead. The means and standard errors (from the unweighted percentages) are sufficient summaries of each column for the present purposes and show that although the percentage of dead diploid spermatozoa is almost three times that of haploids, it is substantially less than $100 \%$, and many diploid rabbit spermatozoa must therefore be alive when ejaculated. The discrepancy between the present (higher) values and those of Beatty \& Fechheimer (1972) is most probably due to the use of formol citrate as a preservative in the present study.

Table 1. Percentages of eosinophilic (presumed dead) haploid and diploid spermatozoa in ejaculates from the AD strain buck over the period of study

\begin{tabular}{|c|c|c|c|}
\hline \multirow[b]{2}{*}{$\begin{array}{l}\text { Ejaculate } \\
\text { sample }\end{array}$} & \multicolumn{2}{|c|}{$\%$ eosinophilic } & \multirow[b]{2}{*}{ Difference } \\
\hline & $\begin{array}{l}\text { Haploid only } \\
(n=1000)\end{array}$ & $\begin{array}{l}\text { Diploid only } \\
(\mathrm{n}=168-226)\end{array}$ & \\
\hline 1 & 6.5 & $33 \cdot 0$ & $-26 \cdot 5$ \\
\hline 2 & 9.0 & $21 \cdot 5$ & $-12 \cdot 5$ \\
\hline 3 & $10 \cdot 0$ & $27 \cdot 0$ & $-17 \cdot 0$ \\
\hline 4 & $12 \cdot 0$ & $29 \cdot 5$ & $-17 \cdot 5$ \\
\hline 5 & $20 \cdot 0$ & $37 \cdot 0$ & $-17 \cdot 0$ \\
\hline 6 & $10 \cdot 5$ & 30.5 & $-20 \cdot 0$ \\
\hline 7 & $21 \cdot 0$ & $36 \cdot 5$ & $-15 \cdot 5$ \\
\hline 8 & $10 \cdot 0$ & $27 \cdot 5$ & $-17 \cdot 5$ \\
\hline 9 & $23 \cdot 5$ & $44 \cdot 0$ & $-20 \cdot 5$ \\
\hline 10 & $10 \cdot 0$ & $49 \cdot 5$ & $-39 \cdot 5$ \\
\hline 11 & $18 \cdot 0$ & $42 \cdot 0$ & $-24 \cdot 0$ \\
\hline 12 & $25 \cdot 5$ & $52 \cdot 5$ & $-27 \cdot 0$ \\
\hline 13 & $15 \cdot 5$ & $37 \cdot 0$ & $-21 \cdot 5$ \\
\hline 14 & $11 \cdot 0$ & $37 \cdot 0$ & $-26 \cdot 0$ \\
\hline 15 & $18 \cdot 0$ & $43 \cdot 0$ & $-25 \cdot 0$ \\
\hline Mean \pm S.E.M. & $14 \cdot 70 \pm 1 \cdot 53$ & $36 \cdot 50 \pm 2 \cdot 23$ & $-21 \cdot 8 \pm 1 \cdot 70$ \\
\hline
\end{tabular}

\section{Survival of ejaculated spermatozoa in vitro}

Linear regressions of the ratios in Table 2 on time (giving equal weight to each ratio) gave fitted lines whose slopes did not differ significantly from zero, showing that diploid rabbit spermatozoa have the same mortality rate as haploids when incubated in these three media. The proportion of live diploid spermatozoa in the ejaculate does not therefore vary for at least $7 \mathrm{~h}$ after ejaculation. 
Table 2. Survival of diploid relative to haploid spermatozoa when incubated in various media at $37^{\circ} \mathrm{C}$

\begin{tabular}{|c|c|c|c|}
\hline \multirow[b]{2}{*}{ Time (h) } & \multicolumn{3}{|c|}{ Percentage relative survival ${ }^{*}$ of diploids in: } \\
\hline & $0.85 \% \mathrm{NaCl}$ & $0.93 \mathrm{BM}$ medium & Hank's BSS \\
\hline 0 & $73 \cdot 8$ & $70 \cdot 4$ & $78 \cdot 4$ \\
\hline 1 & 84.0 & $72 \cdot 4$ & $82 \cdot 6$ \\
\hline 2 & $83 \cdot 4$ & 74.4 & $69 \cdot 1$ \\
\hline 3 & $82 \cdot 4$ & $66 \cdot 5$ & $72 \cdot 6$ \\
\hline 4 & $83 \cdot 1$ & 74.7 & $73 \cdot 3$ \\
\hline 5 & 81.8 & $78 \cdot 1$ & $77 \cdot 7$ \\
\hline 6 & $74 \cdot 1$ & $72 \cdot 4$ & 74.9 \\
\hline 7 & $71 \cdot 7$ & $76 \cdot 9$ & $71 \cdot 8$ \\
\hline Mean \pm S.E. & $79 \cdot 29 \pm 1 \cdot 81$ & $73 \cdot 23 \pm 1 \cdot 31$ & $75.05 \pm 1.53$ \\
\hline Slope \pm S.E. & $-0.813 \pm 0.788$ & $+0.771 \pm 0.529$ & $-0.693 \pm 0.661$ \\
\hline
\end{tabular}

\section{Spermatozoa recovered from the female tract}

The results from nine experiments summarized in Table 3(a), show that at $6 \mathrm{~h}$ post coitum there is a large reduction in the percentage of diploid spermatozoa in the cervix and uterus compared to that in the mean ejaculate. The apparent further fall in the incidence of diploid spermatozoa in the oviducts was not statistically significant $(P>0.05)$. However, a $2 \times 2$ heterogeneity $\chi^{2}$ test on the numbers of diploid and haploid spermatozoa scored from the ejaculates (3554 and 176,446 respectively) and from the oviducts (6 and 2003) showed that the difference in the incidence of diploid spermatozoa is significant at the $0.1 \%$ level.

When the proportions of live and dead spermatozoa recovered from the female tract were scored from nigrosin-eosin preparations, values for the incidence of diploids amongst live spermatozoa only could be estimated for all sources (Table $3 \mathrm{~b}$ ) except the oviducts, because these flushings had at one stage been resuspended in distilled water (see Materials and Methods). These results indicate that the survival of diploid spermatozoa is impaired in the female tract, although it has not been proved that nigrosin-eosin staining is still a valid technique for the differentiation of live and dead spermatozoa after they have been recovered from the female tract.

Observations on spermatozoa with two tails (the incidence and degrees of fusion between the two tails being variable) showed that such spermatozoa were not very successful in traversing, or even penetrating, the cervix (Table 3c), most probably because many of this small population of doubletailed spermatozoa were found to be far less motile than most of those with single tails.

Table 3. Distribution of diploid and double-tailed spermatozoa in the reproductive tract of the female rabbit $6 \mathrm{~h}$ post coitum

\begin{tabular}{lccccc}
\hline & $\begin{array}{c}\text { Mean } \\
\text { ejaculate }\end{array}$ & Vagina & Cervix & Uterus & Oviducts \\
\hline $\begin{array}{l}\text { (a) Diploid spermatozoa (\%) } \\
\text { (b) Live diploid spermatozoa } \\
\text { (as \% of all live sperm.) }\end{array}$ & $1.974 \pm 0.046$ & $1.251 \pm 0.119$ & $0.764 \pm 0.133$ & $0.842 \pm 0.072$ & $0.299 \pm 0.120$ \\
$\begin{array}{l}\text { (c) Double-tailed spermatozoa } \\
\text { (as \% of all sperm.) }\end{array}$ & $1.431 \pm 0.051$ & $1.037 \pm 0.132$ & $0.554 \pm 0.088$ & $0.605 \pm 0.108$ & N.D. \\
$\quad \begin{array}{l}\text { Haploid } \\
\text { Diploid }\end{array}$ & $0.192 \pm 0.015$ & $0.070 \pm 0.015$ & $0.013 \pm 0.006$ & $0.006 \pm 0.004$ & 0.0 \\
& $0.658 \pm 0.030$ & $0.260 \pm 0.045$ & $0.066 \pm 0.012$ & $0.010 \pm 0.005$ & 0.0 \\
\hline
\end{tabular}

Values are means of 9 experiments with standard errors based on discrepancies between experiments, except for diploid spermatozoa in the oviducts, the S.E. of which is based on the expectation of a Poisson distribution for data summed over all experiments. N.D. = not determined. 
Table 4. Relative incidence of diploids among spermatozoa in the uterus and cervix $6 \mathrm{~h}$ post coitum in 9 experiments as the buck aged

\begin{tabular}{ccc}
\hline & $\frac{\%}{\%} 2 \mathrm{~N}$ in uterus & \\
\cline { 2 - 3 } Exp. & $\% 2 \mathrm{~N}$ in cervix & $\begin{array}{c}\text { Age of buck } \\
\text { (weeks) }\end{array}$ \\
\hline 1 & 2.00 & 56 \\
2 & 2.28 & 68 \\
3 & 2.10 & 71 \\
4 & 1.07 & 83 \\
5 & 1.56 & 84 \\
6 & 0.92 & 85 \\
7 & 0.66 & 86 \\
8 & 0.59 & 87 \\
9 & 1.00 & 90 \\
\hline
\end{tabular}

The results presented in Table 4 show how the incidence of diploid spermatozoa in the uterus, relative to that in the cervix, varied between matings. There is a positive correlation $(P<0.001)$ between the ratio and the age of the buck at the time of each experiment.

\section{Discussion}

At ejaculation the percentage of eosinophilic cells (presumed dead) is higher among diploid than haploid spermatozoa (Table 1), but many diploids are alive at that time. After ejaculation diploids and haploids would appear to be equally viable since the relative percentage of live diploids remained unchanged for up to $7 \mathrm{~h}$ in vitro (Table 2), although there is a progressive reduction in the incidence of live diploids as they ascend the female tract (Table 3b). However, if diploid spermatozoa do not contribute to the production of triploid zygotes then some selectivity of sperm transport must be involved to exclude them from the site of fertilization (assuming them to be capable of fertilizing an egg).

The populations of spermatozoa recovered from the higher regions of the female tract have been compared to those of the original ejaculate (i.e. the 'mean ejaculate' results), because it has been shown that the cervical sperm reservoir is built up from the vaginal sperm pool within 5 min of coitus (Bedford, 1971). Therefore the incidence of diploids among the isolated population of superfluous spermatozoa in the vagina $6 \mathrm{~h}$ after coitus cannot be considered as relevant to the distribution of diploids higher in the tract. A large significant reduction in the incidence of diploid spermatozoa has been demonstrated between the ejaculate and the uterus, showing that the cervix is a major barrier to such spermatozoa. Sperm transport through the cervix has been well documented in various species (for reviews see Blandau, 1969, 1973; Bedford, 1972; Mattner, 1973; Moghissi, 1973; Ahlgren, Boström \& Malmqvist, 1974), and it seems that intrinsic sperm motility is the most probable means whereby the majority of spermatozoa penetrate the cervix. There is no evidence that the cervix can 'recognize' or eliminate abnormal spermatozoa, although the physico-chemical structure and properties of cervical mucus (for reviews see Odeblad, 1968, 1973) provide a system in which selection dependent upon sperm motility could take place (see Tampion \& Gibbons, 1962; Morton \& Glover, 1974).

As the buck aged there was a reduction in the ratio of the incidence of diploid spermatozoa in the uterus compared to the cervix (see Table 4). This may indicate a decline with increasing age of the buck's coital performance which would in turn cause inter-mating variability in the induction of a brief phase of rapid sperm transport associated with coitus. The likelihood of alternative explanations must be reduced by the lack of a corresponding trend in the percentage dead among ejaculated diploid spermatozoa over the same period (see Table 1).

Krzanowska (1974) reported that the uterotubal junction was a barrier to presumed diploid mouse spermatozoa, and the present study shows that in the rabbit it effects a reduction in the proportion of diploid spermatozoa among those entering the oviduct. This means that the overall 
incidence of 'live' diploid spermatozoa has been reduced from an initial $1.4 \%$ in the ejaculate to a maximum of $0.3 \%$ of those spermatozoa in the oviducts. This latter value must represent a maximum because it represents the improbable situation of all spermatozoa in the oviducts being alive. These figures reveal a large and highly significant reduction in the incidence of diploid spermatozoa at the site of fertilization ( $6 \mathrm{~h}$ post coitum) compared to that in the ejaculate, although it is insufficient per se to preclude the production of diandric triploids due to the fertilization of normal oocytes by diploid spermatozoa.

This work was supported by a grant from the Ford Foundation and constitutes part of a thesis to be submitted for the degree of Ph.D. to the University of Edinburgh. The author is grateful to his supervisors, Dr R. A. Beatty and Dr T. G. Baker, for their guidance and valuable criticism of the manuscript, and to Mr J. D. Foster for assistance with the animals.

\section{References}

AhLgRen, M., Boström, K. \& MalmQvist, R. (1974) Sperm transport and survival in women with special reference to the Fallopian tube. In Sperm Transport, Survival and Fertilizing Ability in Vertebrates, Vol. 26, pp. 183-200. Eds E. S. E. Hafez \& C. G. Thibault. INSERM, Paris.

BEATTY, R.A. (1964) Density gradient media for mammalian spermatozoa. Proc. 5th Int. Congr. Anim. Reprod. \& A.I., Trento, pp. 276-281.

BeATTY, R.A. (1974) Genetic aspects of spermatozoa. In Physiology and Genetics of Reproduction, pp. 183196. Eds E. M. Coutinho \& F. Fuchs. Basic Life Sciences, vol. 4A. Plenum Press, New York and London.

BeatTy R.A. \& Fechheimer, N.S. (1972) Diploid spermatozoa in rabbit semen and their experimental separation from haploid spermatozoa. Biol. Reprod. 7, 267-277.

BEDFORD, J.M. (1971) The rate of sperm passage into the cervix after coitus in the rabbit. J. Reprod. Fert. 25 , 211-218.

BEDFORD, J.M. (1972) Sperm transport, capacitation and fertilization. In Reproductive Biology, Ch. 6, pp. 338392. Eds H. Balin \& S. R. Glasser. Excerpta Medica, Amsterdam.

Blandau, R.J. (1969) Sperm transport. In The Mammalian Oviduct, pp. 141-162. Eds E. S. E. Hafez \& R. J. Blandau. University Press, Chicago.

Blandau, R.J. (1973) Sperm transport through the mammalian cervix: comparative aspects. In The Biology of the Cervix, Ch. 15, pp. 285-304. Eds R. J. Blandau \& K. S. Moghissi. University Press, Chicago.

Braden, A. W. H. (1953) Distribution of sperms in the genital tract of the female rabbit after ciotus. Aust. J. biol. Sci. 6, 693-705.

Carothers, A.D. \& BeAtTy, R.A. (1975) The recognition and incidence of haploid and polyploid spermatozoa in man, rabbit and mouse. $J$. Reprod. Fert. 44, 487-500.
Casarett, G.W. (1953) A one-solution stain for spermatozoa. Stain Technol. 28, 125-127.

Cohen, J. \& WerretT, D.J. (1975) Antibodies and sperm survival in the female tract of the mouse and rabbit. J. Reprod. Fert. 42, 301-310.

DotT, H.M. \& FosteR, G.C. (1975) Preservation of differential staining of spermatozoa by formol citrate. J. Reprod. Fert. 45, 57-60.

FeChHeIMeR, N.S. \& BEATTY, R.A. (1974) Chromosomal abnormalities and sex ratio in rabbit blastocysts. J. Reprod. Fert. 37, 331-341.

KrZANOWSKA, H. (1974) The passage of abnormal spermatozoa through the uterotubal junction of the mouse. J. Reprod. Fert. 38, 81-90.

MATTNER, P.E. (1973) The cervix and its secretions in relation to fertility. In The Biology of the Cervix, Ch. 18, pp. 339-350. Eds R. J. Blandau \& K. S. Moghissi. University Press, Chicago.

MoghissI, K.S. (1973) Sperm migration through the cervix. In Cervical Mucus in Human Reproduction, pp. 128-152. Eds M. Elstein, K. S. Moghissi \& R. Borth. W.H.O. Colloquium, Geneva, 1972. Scriptor, Copenhagen.

MORTON, D.B. \& GLOVER, T.D. (1974) Sperm transport in the female rabbit: the role of the cervix.J. Reprod. Fert. 38, 131-138.

ODEBLAD, E. (1968) The functional structure of human cervical mucus. Acta obstet. gynec. scand. 47, Suppl. 1, 57-79.

Odeblad, E. (1973) Biophysical techniques of assessing cervical mucus and micro-structure of cervical epithelium. In Cervical Mucus in Human Reproduction, pp. 58-74. Eds M. Elstein, K. S. Moghissi \& R. Borth. W.H.O. Colloquium, Geneva, 1972. Scriptor, Copenhagen.

TAMPION, D. \& GibBONS, R.A. (1962) Orientation of spermatozoa in mucus of the cervix uteri. Nature, Lond. 194, 381. 